\title{
Presupernova neutrinos in large dark matter direct detection experiments
}

\author{
Nirmal Raj, ${ }^{1, *}$ Volodymyr Takhistov, ${ }^{2, \dagger}$ and Samuel J. Witte ${ }^{3, \$}$ \\ ${ }^{1}$ TRIUMF, 4004 Wesbrook Mall, Vancouver, British Columbia V6T 2A3, Canada \\ ${ }^{2}$ Department of Physics and Astronomy, University of California, Los Angeles, \\ Los Angeles, California 90095-1547, USA \\ ${ }^{3}$ Instituto de Física Corpuscular (CSIC-Universitat de València), Paterna (Valencia) E-46980, Spain
}

(Received 6 June 2019; revised manuscript received 11 December 2019; accepted 28 January 2020; published 14 February 2020)

\begin{abstract}
The next Galactic core-collapse supernova (SN) is a highly anticipated observational target for neutrino telescopes. However, even prior to collapse, massive dying stars shine copiously in "pre-supernova" (pre-SN) neutrinos, which can potentially act as efficient SN warning alarms and provide novel information about the very last stages of stellar evolution. We explore the sensitivity to pre-SN neutrinos of large-scale direct dark matter detection experiments, which, unlike dedicated neutrino telescopes, take full advantage of coherent neutrino-nucleus scattering. We find that argon-based detectors with target masses of $\mathcal{O}(100)$ tons (i.e., comparable in size to the proposed ARGO experiment) operating at sub-keV thresholds can detect $\mathcal{O}(10-100)$ pre-SN neutrinos coming from a source at a characteristic distance of $200 \mathrm{pc}$, such as Betelgeuse ( $\alpha$ Orionis). Large-scale xenon-based experiments with similarly low thresholds could also be sensitive to pre-SN neutrinos. For a Betelgeuse-type source, large-scale dark matter experiments could provide a SN warning siren $\sim 10$ hours prior to the explosion. We also comment on the complementarity of large-scale direct dark matter detection experiments and neutrino telescopes in the understanding of core-collapse SN.
\end{abstract}

DOI: 10.1103/PhysRevD.101.043008

\section{INTRODUCTION}

Stars with mass $\gtrsim 8 M_{\odot}$ that ignite nuclear fuel burning nonexplosively explode as core-collapse supernovae ( $\mathrm{SNe}$ ) at the end of their lifetime (see Ref. [1] for a review), leaving behind a compact remnant. As the star's nuclear fuel becomes exhausted, the inner core collapses under gravity. Contraction of the core leads to a dramatic increase in density, with nuclear forces halting and bouncing back the rapid collapse, resulting in a shockwave propagating outward. Copious emission of $\sim 10-30 \mathrm{MeV}$ neutrinos within a $\sim 10 \mathrm{sec}$ burst are a generic byproduct of this event, typically carrying away $\sim 10^{53}$ erg of the original star's gravitational binding energy. The luminosity in neutrinos from core-collapse supernovae greatly exceeds its optical counterpart. The general picture of the outlined supernova mechanism was decisively confirmed by the observation of neutrinos from SN 1987A [2-4]. With the

\footnotetext{
*nraj@triumf.ca

†takhist@physics.ucla.edu

*sam.witte@ific.uv.es
}

Published by the American Physical Society under the terms of the Creative Commons Attribution 4.0 International license. Further distribution of this work must maintain attribution to the author(s) and the published article's title, journal citation, and DOI. Funded by SCOAP. expected rate of Galactic core-collapse $\mathrm{SNe}$ of around a few per century, anticipation and preparation for future SN observations is a principal goal of large-scale neutrino experiments [5]; however, large-scale direct dark matter detection experiments will also be sensitive to $\mathrm{SN}$ neutrinos in a highly complementary manner [6-10].

Even prior to the inception of the core collapse, a significant emission of $\sim \mathrm{MeV}$ "presupernova" (pre-SN) neutrinos is expected from the supernova progenitor during the final nuclear fuel burning stages, in particular from the silicon ( $\mathrm{Si}$ ) burning [11-14]. The resulting pre-SN neutrino luminosity is typically smaller than that of SN neutrinos by a few orders of magnitude. Detection of pre-SN neutrinos will directly probe the very late stages of nuclear fusion processes beyond hydrogen and helium within the SN system, providing vital information about the temperature and density near the star's core at that time. Furthermore, these neutrinos could provide an early supernova warning trigger, dramatically improving upon the current supernova early warning system (SNEWS) [15] network.

Sensitivity studies for pre-SN neutrinos have been conducted [11-14] for current and future neutrino experiments, primarily focusing on liquid scintillator-based (Borexino [16], KamLAND [17,18], SNO+ [19], JUNO $[20,21])$ and water Cherenkov-based (SNO [22], SuperKamiokande [23,24] and Hyper-Kamiokande [25], including gadolinium dissolution [26]) detectors. A dedicated 
study by the KamLAND Collaboration has also been recently carried out [27]. These analyses, however, primarily focused on detection via the inverse beta decay (IBD) $\bar{\nu}_{e}+p \rightarrow e^{+}+n$ channel, which has a kinematic threshold of neutrino energy $=1.8 \mathrm{MeV}$ and is limited to $\bar{\nu}_{e}$ interactions.

Among the major open questions in modern physics is the nature of dark matter (DM). For many decades, a leading DM candidate has been the weakly interacting massive particle (WIMP); however, despite a dedicated and multipronged experimental search program, WIMPs have remained elusive. Some of the most stringent constraints on WIMP DM come from direct detection experiments, which search primarily for rare neutral current interactions with nuclei in deep underground laboratories. Future generations of these experiments will continue to carve out WIMP parameter space, eventually encountering an irreducible background arising from the coherent scattering of neutrinos produced from the Sun, atmospheric interactions, $\mathrm{SNe}$, the interior of the Earth, etc.-collectively, these interactions constitute what is called the "neutrino floor" [28-30]. Since neutrinos can mimic the DM signal, they limit the DM discovery sensitivity. However, the neutrino signal, which definitely exists and will be observed, constitutes in itself an interesting subject of study. It is thus vital to further explore detection capabilities of direct detection experiments beyond particle DM.

Large direct detection experiments are themselves effective neutrino detectors, capable of probing neutrinos in regimes complementary to those studied with conventional neutrino experiments. One reason for this is the very low detection thresholds achievable in direct detection experiments, often around or below the keV level. Furthermore, with heavy nuclei as detector targets, these experiments achieve high detection rates via coherent neutrino-nucleus scattering, whose cross section scales approximately as the neutron number squared. This process has recently been directly observed [31]. Both coherent neutrino-nucleus scattering and elastic neutrino-electron scattering have been considered in a range of studies related to neutrino physics for direct detection experiments, including sterile neutrinos (e.g., Refs. [32,33]), nonstandard neutrino interactions (e.g., Refs. [34,35]), solar neutrinos (e.g., Refs. [33,36-40]), geoneutrinos [41], and neutrinos from DM annihilations and decays [42-44], as well as supernova neutrinos [6-10].

In this work, we explore pre-SN neutrino detection capabilities of large direct detection experiments. Unlike traditional neutrino detectors using IBD, coherent neutrino interactions allow for direct detection experiments to be unconstrained by the IBD kinematic threshold on neutrino energy, and also to have sensitivity to all six $\left(\nu_{e}, \bar{\nu}_{e}, \nu_{\mu}, \bar{\nu}_{\mu}, \nu_{\tau}, \bar{\nu}_{\tau}\right)$ neutrino flavors. As pre-SN neutrinos are significantly softer than $\mathrm{SN}$ neutrinos and also fewer in number, efficient detection entails a low energy threshold and a large target mass. As we will show, a favorable combination of the above is achievable in a future argonbased $\mathcal{O}(100)$ ton-scale detector, such as the recently proposed ARGO experiment [45,46]. Large-scale dark matter direct detection experiments are therefore complementary to neutrino detectors and are insensitive to uncertainties associated with neutrino oscillations.

This paper is organized as follows: In Sec. II, we review the capabilities of future dark matter direct detection experiments, with emphasis on their ability to achieve low energy thresholds suitable for pre-SN neutrino detection. In Sec. III, we show the differential fluxes of pre-SN neutrinos and background neutrinos, and obtain their event rates at direct detection experiments. In Sec. IV, we describe the expected nuclear recoil rates. In Sec. V, we derive our sensitivities to pre-SN neutrinos, showing that an $\mathcal{O}(100)$ ton-scale argon detector (e.g., ARGO $[45,46])$ can constitute an efficient target. In Sec. VI, we summarize and conclude.

\section{LARGE DIRECT DETECTION EXPERIMENTS}

A variety of proposals for the next generation of direct detection experiments has been suggested [47]. As it is difficult to predict the exact final design and hence the associated detection capabilities, we consider optimistic but realistic detector configurations based on current technology. The detector target elements we consider are argon (Ar), xenon (Xe), germanium $(\mathrm{Ge})$, and silicon (Si), which we list in Table I with their respective isotope abundances. ${ }^{1}$

Throughout this work, we optimistically assume that detectors can achieve a perfect detection threshold and energy resolution. Furthermore, we assume that our background originates either from neutrino interactions alone, as might optimistically be the case should conventional backgrounds be reduced to a point where they can be neglected, or from electronic recoils in the detector, which allow us to explore the possibility that reducible backgrounds dominate over those coming from solar neutrinos. The former assumption allows one to treat different experimental configurations on the same footing, independent of specific detector design choices, while the latter allows us to assess the extent to which electronic backgrounds need to be reduced in order to extract useful information from the pre-SN signal. As significant nonneutrino backgrounds are expected, it would be difficult to justify fully neglecting electronic backgrounds for neutrinoelectron scattering signals. For these reasons, and because

\footnotetext{
${ }^{1}$ We do not include isotopic abundances of elements that contribute less than $1 \%$. Note that we refer to naturally occurring abundances, while isotopically modified target material could be used. We do not expect this to significantly alter our conclusions.
} 
TABLE I. Summary of target materials considered for our experimental configurations.

\begin{tabular}{lcc}
\hline \hline Target material & $A(Z)$ & Isotope fraction \\
\hline xenon(Xe) & $128(54)$ & 0.019 \\
& $129(54)$ & 0.264 \\
& $130(54)$ & 0.041 \\
& $131(54)$ & 0.212 \\
& $132(54)$ & 0.269 \\
& $134(54)$ & 0.104 \\
& $136(54)$ & 0.089 \\
argon(Ar) & $40(18)$ & 0.996 \\
germanium(Ge) & $70(32)$ & 0.208 \\
& $72(32)$ & 0.275 \\
& $73(32)$ & 0.077 \\
silicon(Si) & $74(32)$ & 0.363 \\
& $76(32)$ & 0.076 \\
& $28(14)$ & 0.922 \\
& $29(14)$ & 0.047 \\
\hline \hline
\end{tabular}

the neutrino-electron scattering rate is orders of magnitude smaller than the coherent neutrino-nucleus scattering rate (see, e.g., Ref. [8]), we will focus solely on neutrino-nucleus scattering in this work.

We assume our detectors to be located at SNOLAB (Sudbury, Canada), which will likely host a number of next-generation direct detection experiments. However, we emphasize that this assumption will not significantly influence any of our conclusions, as the strong time dependence of the pre-SN neutrino flux allows for unambiguous separation of signal events from the background. The depth of this lab (6010 mwe) ensures that backgrounds due to cosmogenic muons are highly suppressed.

For future large-scale direct detection experiments, we adopt fiducial target masses characteristic of proposed generation-3 experiments and thresholds consistent with those achievable in current and near-future experiments. In particular, we assume that the argon-based experiment will have a detector mass of 300 tons, as proposed for ARGO $[45,46]$. For xenon, we take a detector mass of 50 tons, as proposed for DARWIN [48]. For silicon and germanium, we assume a target mass of $50 \mathrm{~kg}$, similar to what is proposed for SuperCDMS-SNOLAB [49].

We briefly elaborate on the energy thresholds assumed in our analysis. In a recent search for light DM, the argon-based DarkSide-50 experiment has demonstrated the ability to reach a nuclear recoil threshold as low as $0.6 \mathrm{keV}$ while maintaining low backgrounds, although electronic backgrounds in this low-threshold analysis cannot be fully mitigated [50]. ${ }^{2}$ For xenon-based detectors,

\footnotetext{
${ }^{2} \mathrm{~A}$ broader search for DM at DarkSide-50 employs a somewhat higher threshold [51].
}

such as XENON1T [52,53], LUX [54], and PandaX-II [55], typical background-free searches operate efficiently at recoil energies above $\sim 5 \mathrm{keV}$. However, a nuclear recoil threshold of $0.7 \mathrm{keV}$ has recently been achieved [56] at the expense of a reduced efficiency and higher background. For both argon and xenon, these sub-keV thresholds were accomplished using information from only the $S 2$ signal, produced when electrons are drifted out of the liquid and into the gas phase of the time projection chamber (TPC). The drawback of adopting a $S 2$-only analysis is the loss of pulse shape discrimination that is necessary for a strong rejection of electronic recoils. Without any additional experimental techniques, such backgrounds are currently at a level that would plague pre-SN detection. However, there are significant promising experimental $R \& D$ efforts to reduce and better understand the characteristics of these background sources that are currently underway, suggesting that experimental setups with dramatically reduced backgrounds could be feasible by the time generation-3 experiments are operational. Nevertheless, we will present results in Sec. V assuming electronic recoil backgrounds based on measurements from available experiments. We note that the $S 2$-only analyses performed in these experiments typically operate at reduced efficiencies. For example, by comparing the standard analyses performed by DarkSide-50 and Xenon-100 with the $S 2$-only counterpart, the associated efficiencies are only $40 \%$ and $15 \%$, respectively. In order to compare these experiments in a more balanced manner, we neglect this efficiency reduction and simply note that this may affect the observable number of neutrinos in argon and xenon by a factor of $\sim 2$ and $\sim 7$, respectively.

Both germanium and silicon will be used in the future SuperCDMS-SNOLAB experiment. The SuperCDMS Collaboration has recently determined that recoil thresholds as low as $78 \mathrm{eV}$ are achievable with their highvoltage (HV) silicon detectors; however, at the moment their estimation of a $40 \mathrm{eV}$ threshold in the germanium HV detector relies on the use of Lindhard theory and has yet to be directly verified (see Table VII of Ref. [49]). We emphasize that the HV detectors are subject to larger backgrounds than the conventional detectors, with thresholds closer to the level of $\mathcal{O}(200)$ eV [49].

In Table III, we summarize the thresholds and target masses for our experimental configurations. Note that in this table and throughout this work, our threshold values denote the energy deposited, so that $\mathrm{keV}$ denotes $\mathrm{keV}_{\mathrm{r}}$, as opposed to the electron equivalent energy $\left(\mathrm{keV}_{\mathrm{ee}}\right)$. The sensitivities to pre-SN neutrinos of near-future generation-2 experiments, such as the argon-based DarkSide-20k [46] and the xenon-based XENONNT [57] and LZ [58], can be simply inferred by rescaling our results by the corresponding target masses as appropriate. 


\section{PRESUPERNOVA NEUTRINO SIGNAL AND BACKGROUNDS}

\section{A. Presupernova neutrino flux}

When the fuel of a particular element in the core of a star is exhausted, outward thermal pressure forces can no longer counterbalance those induced by gravity. This causes the star to contract. The compression-induced increase in density and temperature can result in the fusion of heavier elements. After nuclear burning within the core, the same element can subsequently burn in the outer shell layers. The later burning stages occur on increasingly shorter timescales. Energy loss can occur from photoemission from the outer surfaces as well as neutrino emission from within the star. After the helium-burning stage, energy losses are dominated by neutrinos produced within the interior. For stars sufficiently massive to reach the silicon-burning stage, the temperatures and densities are so high that electron capture is rampant, resulting in a high flux of $\nu_{e}$ and overall neutronization. This continues until the onset of the core collapse and subsequent shock breakout, which is accompanied by copious neutrino emission.

A variety of thermal and $\beta$ processes contribute to the overall pre-SN neutrino emission, including pair annihilation $\left(e^{+}+e^{-} \rightarrow \nu+\bar{\nu}\right)$, plasmon decays $\left(\gamma^{*} \rightarrow \nu+\bar{\nu}\right)$, photoneutrino production $\left(e^{ \pm}+\gamma \rightarrow e^{ \pm}+\nu+\bar{\nu}\right), e^{ \pm}$capture, and $\beta^{ \pm}$decays. Nonstandard neutrino effects could also affect stellar cooling [59].

The evolution of the pre-SN neutrino flux (compared with the SN neutrino flux) is displayed in Fig. 1, adapted from Ref. [60]. While the prediction of the pre-SN neutrino flux is not without modeling uncertainties, independent numerical simulations have found closely matching results. These uncertainties are subdominant compared to the

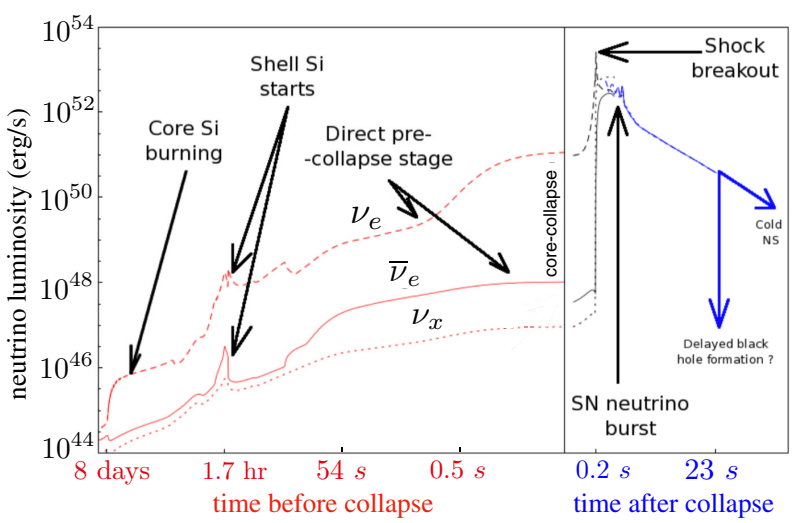

FIG. 1. Evolution of the neutrino luminosity of a $15 M_{\odot}$ star in the final stages of its life. Contributions from electron neutrinos $\left(\nu_{e}\right)$, electron antineutrinos $\left(\bar{\nu}_{e}\right)$, and the averaged contribution from the $\nu_{\mu}, \bar{\nu}_{\mu}, \nu_{\tau}$, and $\bar{\nu}_{\tau}$ flavors (denoted by " $\nu_{x}$ ") are shown, for both before supernova core collapse (pre-SN neutrinos) and after core collapse (SN neutrinos). Note the change of scale on the $x$ axis after core collapse. This figure is adapted from Ref. [60]. uncertainties on the measurements of the distances to source stars. For our analysis, we use the tabulated neutrino fluxes for $M=15 M_{\odot}$ and $M=30 M_{\odot}$ stars as computed by Patton et al. [14]. We have confirmed that using the fluxes computed by Odrzywolek et al. [11] does not significantly alter our results.

\section{B. Neutrino backgrounds}

Since pre-SN neutrinos carry $\sim \mathrm{MeV}$ energies, the relevant backgrounds are due to solar, reactor and geoneutrinos (see Fig. 2), which dominate at energies $E_{\nu} \lesssim 10 \mathrm{MeV}$. Among these, reactor and geoneutrino fluxes depend sensitively on the detector location. We have summarized these backgrounds in Table II. Although our main background originates from solar ${ }^{8} \mathrm{~B}$ neutrinos, which has significant flux even for neutrino energies beyond $10 \mathrm{MeV}$, we also comment on the other background contributions for completeness.

\section{Solar neutrinos}

Electron neutrinos that are produced via nuclear fusion reactions in the Sun contribute predominantly to the neutrino background at low energies (see Ref. [61] for a review). This background is independent of the laboratory location. The solar neutrino flux is composed of contributions from multiple reaction chains, with varying resultant fluxes and energies. The proton-proton cycle contributes to more than $98 \%$ of the energy flux. Initiated by the reaction $p+p \rightarrow{ }^{2} \mathrm{H}+e^{+}+\nu_{e}$, this cycle gives rise to $p p$, hep, pep, ${ }^{7} \mathrm{Be}$, and ${ }^{8} \mathrm{~B}$ neutrinos. The carbon-nitrogen-oxygen (CNO) cycle accounts for the rest of the Sun's energy, giving rise to ${ }^{13} \mathrm{~N},{ }^{15} \mathrm{O}$, and ${ }^{17} \mathrm{~F}$ neutrinos.

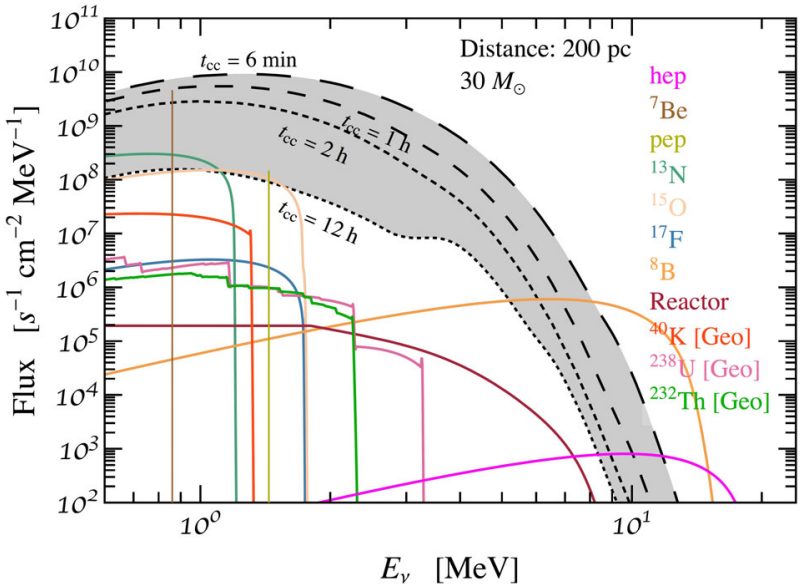

FIG. 2. Neutrino fluxes at the SNOLAB location. The presupernova neutrino signal flux (dashed curves) is evaluated at various times $t_{c c}$ prior to core collapse. The backgrounds (solid curves) originate from solar, reactor, and geo-neutrinos. Their fluxes and maximum energies are provided in Table II. The solar ${ }^{8} \mathrm{~B}$ neutrinos constitute our main background. 
TABLE II. Fluxes and maximum energies of the neutrino backgrounds to the presupernova neutrino signal in direct detection experiments. These consist of solar, reactor, and geoneutrino contributions. The solar ${ }^{8} \mathrm{~B}$ neutrinos constitute our main background.

\begin{tabular}{llc}
\hline \hline & & Max energy \\
Neutrino component & Flux $\left[\mathrm{cm}^{-2} \mathrm{~s}^{-1}\right]$ & $E_{\nu}[\mathrm{MeV}]$ \\
\hline Solar $\left(\nu_{\mathrm{e}}, p p\right)$ & $6.03(1 \pm 0.006) \times 10^{10}$ & 0.42 \\
Solar $\left(\nu_{\mathrm{e}}\right.$, pep $[$ line] $\left.]\right)$ & $1.47(1 \pm 0.012) \times 10^{8}$ & 1.45 \\
Solar $\left(\nu_{\mathrm{e}}\right.$, hep $)$ & $8.31(1 \pm 0.300) \times 10^{3}$ & 18.77 \\
Solar $\left(\nu_{\mathrm{e}},{ }^{7} \mathrm{Be}[\right.$ line 1]) & $4.56(1 \pm 0.070) \times 10^{8}$ & 0.39 \\
Solar $\left(\nu_{\mathrm{e}},{ }^{7} \mathrm{Be}[\right.$ line 2$\left.]\right)$ & $4.10(1 \pm 0.070) \times 10^{9}$ & 0.87 \\
Solar $\left(\nu_{e},{ }^{8} \mathrm{~B}\right)$ & $4.59(1 \pm 0.140) \times 10^{6}$ & 16.80 \\
Solar $\left(\nu_{e},{ }^{13} \mathrm{~N}\right)$ & $2.17(1 \pm 0.140) \times 10^{8}$ & 1.20 \\
Solar $\left(\nu_{e},{ }^{15} \mathrm{O}\right)$ & $1.56(1 \pm 0.150) \times 10^{8}$ & 1.73 \\
Solar $\left(\nu_{e},{ }^{17} \mathrm{~F}\right)$ & $3.40(1 \pm 0.170) \times 10^{6}$ & 1.74 \\
Reactor $\left(\bar{\nu}_{\mathrm{e}}\right)$ & $5.96(1 \pm 0.080) \times 10^{5}$ & 10.00 \\
Geo $\left(\bar{\nu}_{e},{ }^{40} \mathrm{~K}\right)$ & $2.19(1 \pm 0.168) \times 10^{7}$ & 1.32 \\
Geo $\left(\bar{\nu}_{e},{ }^{238} \mathrm{U}\right)$ & $4.90(1 \pm 0.200) \times 10^{6}$ & $3.99^{\mathrm{a}}$ \\
Geo $\left(\bar{\nu}_{e},{ }^{232} \mathrm{Th}\right)$ & $4.55(1 \pm 0.257) \times 10^{6}$ & 2.26 \\
\hline \hline
\end{tabular}

${ }^{a}$ In Fig. 2, the ${ }^{238} \mathrm{U}$ flux appears to cut off at much lower energies; however, this is simply a consequence of not extending the $y$ axis to lower values. See Fig. 1 of Ref. [30] for an extended spectrum.

Predictions of solar neutrino fluxes depend on the solar model. The Standard Solar Model (GS98) [62] has been demonstrated to agree well with helioseismological studies. However, more modern models such as AGSS09 [63], that are more internally consistent, show a lesser degree of agreement with the helioseismology results, leading to a discrepancy known as the "solar metallicity" problem. In this work, we will assume the solar neutrino fluxes and uncertainties as predicted by AGSS09 (see Table 2 of Ref. [61]). These are provided in Table II. Our dominant background originates from ${ }^{8} \mathrm{~B}$ neutrinos. Since the ${ }^{8} \mathrm{~B}$ flux difference between the two solar models is only $\sim 20 \%$, using a different solar model will not significantly affect our results. Future measurements of the solar neutrino flux will further reduce this uncertainty.

\section{Reactor neutrinos}

Fission $\beta$ decays of uranium $\left({ }^{235} \mathrm{U}\right.$ and $\left.{ }^{238} \mathrm{U}\right)$ and plutonium $\left({ }^{239} \mathrm{Pu}\right.$ and $\left.{ }^{241} \mathrm{Pu}\right)$ in reactor fuels give rise to reactor electron antineutrinos (see Ref. [64] for a review). The corresponding neutrino flux depends sensitively on the reactor operation since these isotopes are short-lived, and since the reactor's fuel composition and relative isotope fractions evolve with time. The reactor neutrino background depends on the location of the direct detection experiment-i.e., on the specifics of and distances to nearby reactors. For the SNOLAB location, we calculate this background using the formalism and reactors described in Ref. [30].

\section{Geoneutrinos}

Geoneutrinos are predominantly electron antineutrinos ${ }^{3}$ originating from the $\beta$-decay branches of the Earth's major heat-producing nuclear reactions, involving isotopes of potassium $\left({ }^{40} \mathrm{~K}\right)$, thorium $\left({ }^{232} \mathrm{Th}\right)$, and uranium $\left({ }^{238} \mathrm{U}\right)$. Recently, KamLAND [65] and Borexino [66] have observed a geoneutrino flux. We take the spectrum for each of these elements from Ref. [65]. The respective location-dependent total flux is predicted from a geophysics-based three-dimensional global Earth model of heat-producing element distribution [67].

\section{NUCLEAR RECOIL RATES}

The Standard Model neutrino-nucleus coherent scattering cross section is given by [68]

$$
\frac{d \sigma^{I}}{d E_{r}}\left(E_{\nu}, E_{r}\right)=\frac{G_{F}^{2} m_{I}}{4 \pi} Q_{W}^{2}\left(1-\frac{m_{I} E_{r}}{2 E_{\nu}^{2}}\right) F_{I}^{2}\left(E_{r}\right),
$$

where $m_{I}$ is the target nuclide mass; $G_{F}=1.1664 \times$ $10^{-5} \mathrm{GeV}^{-2}$ is the Fermi coupling constant; $F_{I}\left(E_{r}\right)$ is the form factor, taken to be the Helm form factor [69]; $Q_{W}=\left[\left(1-4 \sin ^{2} \theta_{\mathrm{W}}\right) Z_{I}-N_{I}\right]$ is the weak nuclear charge; $N_{I}$ is the number of neutrons; $Z_{I}$ is the number of protons; and $\theta_{\mathrm{W}}$ is the Weinberg angle. Since $\sin ^{2} \theta_{\mathrm{W}}=0.23867$ at low energies [70], the coefficient in front of $Z_{I}$ in $Q_{W}$ approximately vanishes, and the coherent neutrino-nucleus scattering cross section follows an approximate $N_{I}^{2}$ scaling.

For a differential neutrino flux $d \phi_{\nu} / d E_{\nu}$, the differential event rate per unit time per unit detector mass is given by

$$
\frac{d R_{\nu}^{I}}{d E_{r}}\left(E_{r}\right)=\frac{C_{I}}{m_{I}} \int_{E_{\nu}^{\min }}^{\infty} \frac{d \phi_{\nu}}{d E_{\nu}} \frac{d \sigma^{I}}{d E_{r}}\left(E_{\nu}, E_{r}\right) d E_{\nu},
$$

where $E_{\nu}^{\min }$ is the minimum neutrino energy required to produce a recoil of energy $E_{r}$, given by

$$
E_{\nu}^{\min }=\sqrt{\frac{m_{I} E_{r}}{2}} .
$$

Note that the maximum recoil energy due to collision with a neutrino of energy $E_{\nu}$ is

$$
E_{r}^{\max }=\frac{2 E_{\nu}^{2}}{m_{I}+2 E_{\nu}} .
$$

In Eq. (2), $C_{I}$ is the mass fraction of nuclide $I$ in the material. When multiple nuclides are present, the differential event rate is obtained by combining their contributions. The total event rate over the exposure of the experiment is

\footnotetext{
${ }^{3}$ We note that electron neutrinos are also produced in subdominant quantities (e.g., from electron capture in ${ }^{40} \mathrm{~K}$, contributing at the level of $\sim 11 \%$ ). We neglect this contribution here, as geoneutrinos are not expected to noticeably impede the detection of pre-SN neutrinos.
} 


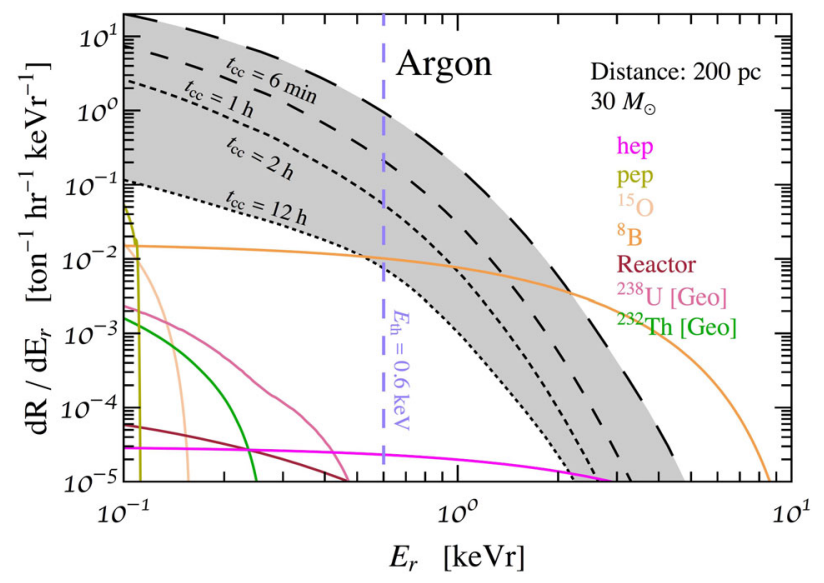

FIG. 3. Predicted nuclear recoil spectra for a pre-SN neutrino signal in argon for various time intervals until the onset of the core collapse, assuming a characteristic $30 M_{\odot}$ star located at a distance of 200 pc. Background contributions from solar, reactor, and geoneutrinos are also shown for comparison.

$$
\frac{d R_{\nu}}{d E_{r}}=M \int d T \sum_{I} \frac{d R_{\nu}^{I}}{d E_{r}}
$$

where $M$ is the fiducial target mass of the detector, and the integration $\int d T$ runs over the data-taking period. For neutrino fluxes that are independent of time, these factors can be combined into a single multiplicative term MT that accounts for the total exposure of the experiment.

Since coherent neutrino-nucleus scattering is mediated by the $Z$ boson, this detection channel is independent of neutrino flavor and the corresponding oscillation effects. Oscillation effects do become relevant if neutrino-electron scattering is considered [41].

We display the expected pre-SN neutrino signal as well as background nuclear recoil spectra for a 300 ton argon experiment (i.e., ARGO-type detector) in Fig. 3. The pre-SN neutrino signal is evaluated at several time intervals prior to the onset of core collapse, assuming a characteristic $30 M_{\odot}$ star located at a distance of $200 \mathrm{pc}$. Already around $12 \mathrm{~h}$ prior to core collapse, the differential recoil rate of the pre-SN neutrino signal near the $0.6 \mathrm{keV}$ detection threshold starts to exceed that of background ${ }^{8} \mathrm{~B}$ solar neutrinos.

\section{DETECTION SENSITIVITY}

A burst of SN neutrinos is readily detectable with large direct detection experiments for a SN located $\mathcal{O}(10) \mathrm{kpc}$ away (i.e., Milky Way distance scales) [6-10]. While argon detectors have not been previously explored as targets for SN neutrinos, the resulting sensitivity can be expected to be similar to xenon-based configurations. In contrast to SN neutrinos, the pre-SN signal is fainter, and the detection distance can be expected to be on $\lesssim 1 \mathrm{kpc}$ scales. Nearby red supergiant stars at the end of their lifetime constitute the most likely source for pre-SN neutrino emission. There are 41 red supergiant stars with distance estimates within $1 \mathrm{kpc}$, 16 within $0.5 \mathrm{kpc}$, and 5 within $0.2 \mathrm{kpc}$ (see Table 2 of Ref. [71]). The most well studied is the red supergiant Betelgeuse ( $\alpha$ Orionis), with mass $17-25 M_{\odot}$ and distance ${ }^{4}$ $197 \pm 45$ pc [74], which we take as our characteristic source.

We estimate our detection sensitivities in two ways. First, we compute the cumulative number of pre-SN neutrino events integrated from some time $t=t_{c c}$ prior to core collapse, all the way to core collapse, $t=0$ :

$$
N_{\text {events }}\left(t_{c c}\right) \equiv M \times \int_{-t_{c c}}^{0} d T \int d E_{r} \frac{d R_{\nu}}{d E_{r}}
$$

This quantity is displayed on the left-hand panel of Fig. 4 for two stellar masses, $M=15 M_{\odot}$ and $M=30 M_{\odot}$ (shown in solid curves). Also shown in Fig. 4 using dashed lines is the ratio of the number of signal (or pre-SN) events $N_{\mathrm{SN}}$ to the square root of the total number of events $\left(N_{\mathrm{SN}}+N_{\mathrm{bkg}}\right)$ as a function of $t_{c c}$, assuming a distance of $200 \mathrm{pc}$ and a ${ }^{8} \mathrm{~B}$ flux given by the expected mean. It is worth emphasizing that this statistic neglects valuable information on the time dependence of the signal (i.e., searches for time-correlated events in a particular window) which could be used to better extract the signal for small $t_{c c}$. The $t_{c c}$ values are chosen to conveniently illustrate the typical average flux behavior. It can be seen that $\mathcal{O}(10)$ pre-SN neutrino events are expected $\sim 1$ day prior to the $\mathrm{SN}$ explosion and would exceed the ${ }^{8} \mathrm{~B}$ neutrino background. This quantity can be extracted from the dataset after the occurrence of the $\mathrm{SN}$, providing a valuable window into the last stages of stellar evolution.

A second useful quantity is the expected number of pre-SN neutrino events obtained in an interval starting $t=12 \mathrm{~h}$ prior to core collapse and ending at some value $t=t_{c c}$ :

$$
\Delta N_{12 h \rightarrow t_{c c}}^{\mathrm{evts}}\left(t_{c c}\right) \equiv M \times \int_{-12 \mathrm{~h}}^{-t_{c c}} d T \int d E_{r} \frac{d R_{\nu}}{d E_{r}} .
$$

This is shown in the right-hand panel of Fig. 4. This quantity tracks the number of events accumulated in real time and provides a direct visualization of the rising fluence of pre-SN neutrinos. In practice, this event-tracking scheme may be implemented within the experiment by registering events over 12-hour windows, with new windows starting every few minutes. ${ }^{5}$ Also shown in the figure are the $1 \sigma$ and $3 \sigma$

\footnotetext{
${ }^{4}$ We note that recent parallax measurements obtained from Gaia data [72] could further decrease the uncertainty in distances to nearby red supergiants (see, e.g., Ref. [73]).

${ }^{5}$ This simple proposal of how event tracking could be implemented may be in some conflict with the currently applied blinding schemes for dark matter searches, in which the region of interest is often excluded from the analysis until unblinding.
} 

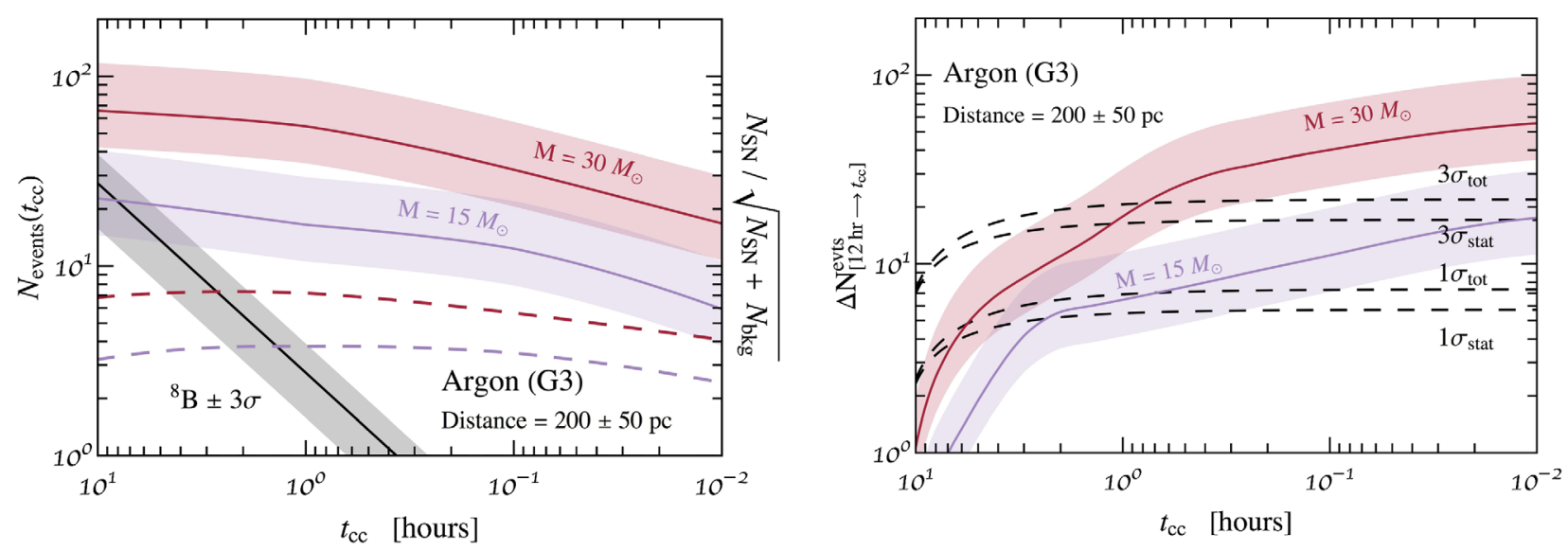

FIG. 4. Expected number of presupernova neutrino events above detection threshold in a generation-3 liquid argon-based dark matter direct detection experiment of target mass 300 tons. These are shown for stellar masses of $15 M_{\odot}$ and $30 M_{\odot}$ for a star at a distance scale of $200 \mathrm{pc}$, such as Betelgeuse. The error band on the presupernova neutrino signal is the $\pm 1 \sigma$ flux uncertainty due to the uncertainty in the star's location. The errors on the background ${ }^{8} \mathrm{~B}$ neutrinos come from the systematic uncertainty in the neutrino flux (see Table II). Left: Shown in solid curves are the number of events integrated from the time until core collapse, $t=-t_{c c}$, to the point of core collapse, $t=0$; shown in dashed curves are the ratios of pre-SN events $N_{\mathrm{SN}}$ to the square root of $N_{\mathrm{SN}}+N_{\mathrm{bkg}}$, assuming a SN distance of 200 pc and that the ${ }^{8} \mathrm{~B}$ flux is given by the expected mean value. Right: The number of events integrated from 12 hours before core collapse, $t=-12 \mathrm{~h}$, to the time until core collapse, $t=t_{c c}$. Also shown here are $1 \sigma$ and $3 \sigma$ statistical and total (statistical and systematic) fluctuations of the ${ }^{8} \mathrm{~B}$ neutrino background.
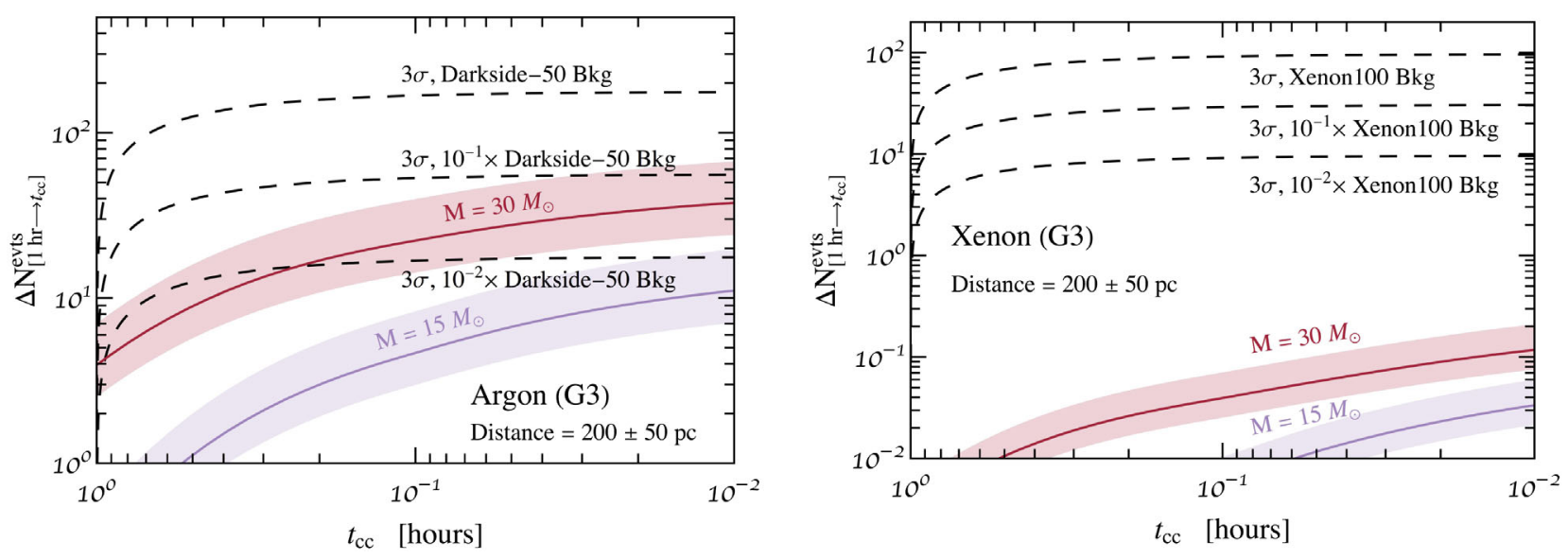

FIG. 5. Expected number of presupernova neutrino events above the detection threshold in generation-3 dark matter direct detection experiments with a liquid argon target of mass 300 tons (left) and with a liquid xenon target of mass 50 tons (right). These are shown for stellar masses of $15 M_{\odot}$ and $30 M_{\odot}$ for a star at a distance of $200 \mathrm{pc}$. Also shown are $3 \sigma$ fluctuations on electronic recoil backgrounds estimated from measurements in Ref. [50,56] for argon and xenon, respectively (see text for further details). The error band on the presupernova neutrino signal is the $\pm 1 \sigma$ flux uncertainty due to the uncertainty in the star's location.

statistical and total (statistical plus systematic) fluctuations of the solar ${ }^{8} \mathrm{~B}$ neutrino background. Note that statistical and systematic uncertainties in the ${ }^{8} \mathrm{~B}$ flux are comparable for $\Delta N_{12 h \rightarrow t_{c c}}^{\text {evts }}(0)$, and thus integrating over a smaller time interval [e.g., instead choosing to analyze, say, $\Delta N_{6 h \rightarrow t_{c c}}^{\mathrm{evts}}(0)$ ] tends to produce subdominant statistical fluctuations. Already at $\mathcal{O}(10)$ hours prior to collapse, the pre-SN neutrino signal will be visible at a $3 \sigma$ level above the background for a $M=30 M_{\odot}$ star. For $M=15 M_{\odot}$, this observation threshold is crossed within an hour of collapse.
We note that our projections are conservative, and a better future understanding of the ${ }^{8} \mathrm{~B}$ solar neutrino background will improve the signal sensitivity. ${ }^{6}$

In Fig. 5, we display $\Delta N_{1 h \rightarrow t_{c c}}^{\text {evts }}\left(t_{c c}\right)$, defined analogously to $\Delta N_{12 h \rightarrow t_{c c}}^{\text {evts }}\left(t_{c c}\right)$, along with $3 \sigma$ fluctuations associated

\footnotetext{
${ }^{6} \mathrm{~A}$ reduction of ${ }^{8} \mathrm{~B}$ flux uncertainty by a factor of 2 compared to what we used in our study will allow us to detect pre-SN events above $3 \sigma$ fluctuations of the ${ }^{8} \mathrm{~B}$ background significantly earlier, at $t_{c c}=1.5$ hours, rather than the displayed $t_{c c}=0.05$ hours.
} 
TABLE III. Observational prospects for a presupernova neutrino signal originating from $15 M_{\odot}$ and $30 M_{\odot}$ stars in future large direct dark matter detection experiments with configurations suggested by currently proposed detectors, based on argon (ARGO), xenon (DARWIN), germanium, and silicon (both SuperCDMS-SNOLAB), with their respective experimental fiducial mass and threshold. The expected number of presupernova neutrino events within a 12-hour time window prior to the collapse for a SN 200 pc away from Earth and the distance to the source that corresponds to a single mean predicted number of events are displayed. We note that these results are sensitive to an assumed level of contributing background.

\begin{tabular}{|c|c|c|c|c|c|c|c|}
\hline Target & $\begin{array}{l}\text { Mass } \\
\text { [tons] }\end{array}$ & $\begin{array}{c}\text { Threshold } \\
{\left[\mathrm{keV}_{r}\right]}\end{array}$ & Ref. & $\begin{array}{c}N_{\text {events }}(12 \mathrm{~h}) \\
15 M_{\odot} \\
{[d=200 \mathrm{pc}]}\end{array}$ & $\begin{array}{c}d_{\mathrm{SN}}(N=1) \\
15 M_{\odot} \\
{[\mathrm{pc}]}\end{array}$ & $\begin{array}{c}N_{\text {events }}(12 \mathrm{~h}) \\
30 M_{\odot} \\
{[d=200 \mathrm{pc}]}\end{array}$ & $\begin{array}{c}d_{\mathrm{SN}}(N=1) \\
30 M_{\odot} \\
{[\mathrm{pc}]}\end{array}$ \\
\hline argon-G2 & 20 & 0.6 & [46] & 1.53 & 251 & 4.56 & 427 \\
\hline argon & 300 & 0.6 & {$[45,46]$} & 23.6 & 972 & 68.4 & 1654 \\
\hline xenon-G2 & 7 & 0.7 & {$[56,57,75]$} & 0.013 & 23.1 & 0.041 & 40.5 \\
\hline xenon & 50 & 0.7 & {$[48,56]$} & 0.095 & 61.8 & 0.292 & 108 \\
\hline germanium & 0.05 & 0.04 & [49] & 0.160 & 78 & 0.356 & 119 \\
\hline silicon & 0.05 & 0.08 & [49] & 0.054 & 46.5 & 0.123 & 70 \\
\hline
\end{tabular}

with the possible electron recoil backgrounds in generation-3 argon and xenon detectors. We estimate these backgrounds based on measurements by the argon-based Darkside-50 [50] and xenon-based XENON100 [56] experiments. Since electronic recoils can be large at energies above those relevant for pre-SN neutrinos, we compute these background rates using an upper limit on the recoil energy of 1.7 and $2 \mathrm{keVnr}$ for xenon and argon, respectively. Over the energy range of interest, these rates are approximately independent of recoil energy, being $\sim 0.2$ (argon) and $\sim 0.5$ (xenon) events $/ \mathrm{keVnr} / \mathrm{kg} /$ day. We consider the more pessimistic case where these backgrounds are not reduced when generation-3 experiments come online, as well as the more optimistic one where they are reduced by a factor of 10 or 100 at such a time.

In Table III, we list the expected number of observed pre-SN neutrino events within a 12-hour period prior to the collapse, and also the distance from the source at which a single event is observable, for the proposed large-scale direct detection configurations we consider. Due to a potentially larger achievable target mass and lower threshold, an argon-based experiment could be more sensitive to pre-SN neutrinos than an experiment based on xenon. Despite potentially lower thresholds for germanium- and silicon-based configurations, we expect that an argon-based experiment could constitute a significantly more favorable pre-SN neutrino detector due to the much greater target mass.

We note that by significantly improving the detection threshold for a given target material, similar event rates can be achieved with smaller exposures. In Fig. 6, we illustrate how the sensitivity depends on the adopted threshold (taking the maximum recoil energy to be $10 \mathrm{keV}$ ) for each of our experimental configurations, for a $15 M_{\odot}$ (left) and a $30 M_{\odot}$ (right) star at $200 \mathrm{pc}$. We display here the number of events per unit target mass (in units of ton ${ }^{-1}$ ) to allow for a straightforward comparison between experiments.
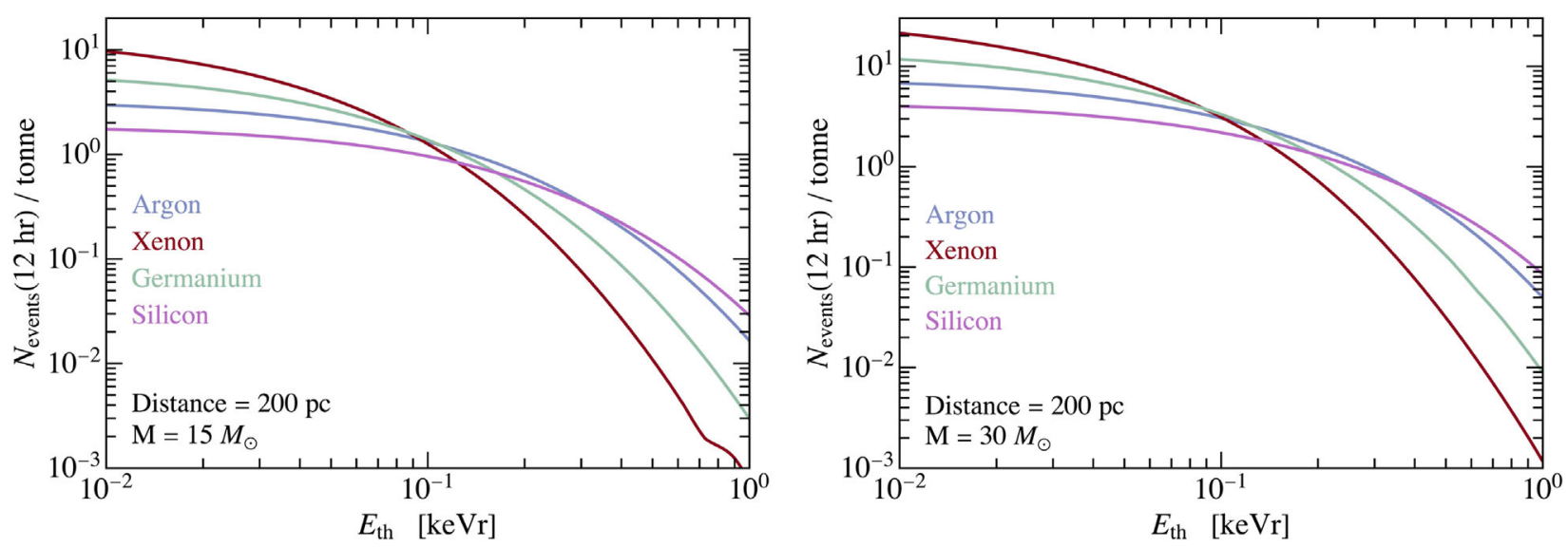

FIG. 6. Expected number of events per unit target mass as a function of energy threshold, for detectors with argon, xenon, germanium, and silicon, assuming a $15 M_{\odot}$ (left) and $30 M_{\odot}$ (right) star at a distance of $200 \mathrm{pc}$. 


\section{SUMMARY}

A future supernova is a highly anticipated event, and experiments that can detect the corresponding presupernova neutrinos can both serve as efficient supernova alarm triggers and provide novel insights into the final stages of the lifetime of massive stars. Future large-scale dark matter direct detection experiments can also act as effective neutrino telescopes. In this work we have explored their sensitivity to presupernova neutrino signal detection.

Unlike conventional neutrino experiments that primarily employ inverse beta decay for SN-related neutrinos, direct detection experiments will detect these neutrinos primarily through the channel of coherent nuclear scattering, which has many advantages. First, the event rate is independent of neutrino mass hierarchy and oscillations, and in particular there is no penalty to pay in the case of inverted hierarchy [27]. Second, since all neutrino flavors participate in coherent nuclear scattering, the presupernova neutrino flux that could be detected in direct detection experiments is potentially larger compared to typical neutrino experiments. We do note, however, that the direct detection experiments we consider are insensitive to the directionality of the presupernova neutrinos. ${ }^{7}$

\footnotetext{
${ }^{7}$ However, directionality may be challenging for neutrino experiments as well, due to IBD detection techniques, as well as the low energies of presupernova neutrinos.
}

In summary, we have shown that large-scale dark matter direct detection experiments, especially those based on argon and xenon, can constitute promising presupernova neutrino detectors. Large-scale direct detection experiments thus complement dedicated neutrino telescopes as presupernova detectors. Our work also highlights the necessity for further efforts to reduce experimental backgrounds at lower thresholds in direct detection experiments that will allow us to open sensitivity for new programs of research.

\section{ACKNOWLEDGMENTS}

We would like to thank Joe Bramante, Michela Lai, Rafael Lang, Ingrida Semenec, Michael Smy, Hank Sobel, Mark Vagins, and Tien-Tien Yu for helpful discussions. The work of N.R. is supported by the Natural Sciences and Engineering Research Council of Canada; TRIUMF receives federal funding via a contribution agreement with the National Research Council Canada. The work for V.T. was supported by the U.S. Department of Energy (DOE) Grant No. DESC0009937. S. J.W. acknowledges support from Spanish MINECO national Grants No. FPA201457816-P and No. FPA2017-85985-P, and from the European Projects No. H2020-MSCAITN-2015// 674896-ELUSIVES and No. H2020-MSCA-RISE2015.
[1] A. Burrows, Rev. Mod. Phys. 85, 245 (2013).

[2] R. M. Bionta et al., Phys. Rev. Lett. 58, 1494 (1987).

[3] K. Hirata et al. (Kamiokande-II Collaboration), Phys. Rev. Lett. 58, 1490 (1987).

[4] E. N. Alekseev, L. N. Alekseeva, I. V. Krivosheina, and V. I. Volchenko, Phys. Lett. B 205, 209 (1988).

[5] A. Mirizzi, I. Tamborra, H.-T. Janka, N. Saviano, K. Scholberg, R. Bollig, L. Hudepohl, and S. Chakraborty, Riv. Nuovo Cimento 39, 1 (2016).

[6] S. Chakraborty, P. Bhattacharjee, and K. Kar, Phys. Rev. D 89, 013011 (2014).

[7] K. Abe et al. (XMASS Collaboration), Astropart. Phys. 89, 51 (2017).

[8] R. F. Lang, C. McCabe, S. Reichard, M. Selvi, and I. Tamborra, Phys. Rev. D 94, 103009 (2016).

[9] T. Kozynets, S. Fallows, and C. B. Krauss, Astropart. Phys. 105, 25 (2019).

[10] D. Khaitan (LZ Collaboration), J. Inst. 13, C02024 (2018).

[11] A. Odrzywolek, M. Misiaszek, and M. Kutschera, Astropart. Phys. 21, 303 (2004).

[12] C. Kato, M. D. Azari, S. Yamada, K. Takahashi, H. Umeda, T. Yoshida, and K. Ishidoshiro, Astrophys. J. 808, 168 (2015).
[13] T. Yoshida, K. Takahashi, H. Umeda, and K. Ishidoshiro, Phys. Rev. D 93, 123012 (2016).

[14] K. M. Patton, C. Lunardini, R. J. Farmer, and F. X. Timmes, Astrophys. J. 851, 6 (2017).

[15] P. Antonioli et al., New J. Phys. 6, 114 (2004).

[16] G. Alimonti et al. (Borexino Collaboration), Nucl. Instrum. Methods Phys. Res., Sect. A 600, 568 (2009).

[17] A. Gando et al. (KamLAND Collaboration), Phys. Rev. D 88, 033001 (2013).

[18] A. Gando et al. (KamLAND Collaboration), Phys. Rev. C 92, 055808 (2015).

[19] S. Andringa et al. (SNO+Collaboration), Adv. High Energy Phys. 2016, 6194250 (2016).

[20] F. An et al. (JUNO Collaboration), J. Phys. G 43, 030401 (2016).

[21] R. Han, Y.-F. Li, L. Zhan, W. F. McDonough, J. Cao, and L. Ludhova, Chin. Phys. C, 40, 033003 (2016).

[22] B. Aharmim et al. (SNO Collaboration), Phys. Rev. C 88, 025501 (2013).

[23] Y. Fukuda et al. (Super-Kamiokande Collaboration), Nucl. Instrum. Methods Phys. Res., Sect. A 501, 418 (2003).

[24] K. Abe et al., Nucl. Instrum. Methods Phys. Res., Sect. A 737, 253 (2014). 
[25] K. Abe et al. (Hyper-Kamiokande Collaboration), arXiv: 1805.04163.

[26] J. F. Beacom and M. R. Vagins, Phys. Rev. Lett. 93, 171101 (2004).

[27] K. Asakura et al. (KamLAND Collaboration), Astrophys. J. 818, 91 (2016).

[28] J. Billard, L. Strigari, and E. Figueroa-Feliciano, Phys. Rev. D 89, 023524 (2014).

[29] J. Monroe and P. Fisher, Phys. Rev. D 76, 033007 (2007).

[30] G. B. Gelmini, V. Takhistov, and S. J. Witte, J. Cosmol. Astropart. Phys. 07 (2018) 009.

[31] D. Akimov et al. (COHERENT Collaboration), Science 357, 1123 (2017).

[32] M. Pospelov, Phys. Rev. D 84, 085008 (2011).

[33] J. Billard, L. E. Strigari, and E. Figueroa-Feliciano, Phys. Rev. D 91, 095023 (2015).

[34] R. Harnik, J. Kopp, and P. A. N. Machado, J. Cosmol. Astropart. Phys. 07 (2012) 026.

[35] B. Dutta, S. Liao, L. E. Strigari, and J. W. Walker, Phys. Lett. B 773, 242 (2017).

[36] M. Schumann, L. Baudis, L. Butikofer, A. Kish, and M. Selvi, J. Cosmol. Astropart. Phys. 10 (2015) 016.

[37] D. Franco et al., J. Cosmol. Astropart. Phys. 08 (2016) 017.

[38] D. G. Cerdeno, J. H. Davis, M. Fairbairn, and A. C. Vincent, J. Cosmol. Astropart. Phys. 04 (2018) 037.

[39] R. Essig, M. Sholapurkar, and T.-T. Yu, Phys. Rev. D 97, 095029 (2018).

[40] J. L. Newstead, L. E. Strigari, and R. F. Lang, Phys. Rev. D 99, 043006 (2019).

[41] G. B. Gelmini, V. Takhistov, and S. J. Witte, Phys. Rev. D 99, 093009 (2019).

[42] J. F. Cherry, M. T. Frandsen, and I. M. Shoemaker, Phys. Rev. Lett. 114, 231303 (2015).

[43] Y. Cui, M. Pospelov, and J. Pradler, Phys. Rev. D 97, 103004 (2018).

[44] D. McKeen and N. Raj, Phys. Rev. D 99, 103003 (2019).

[45] G. Zuzel, P. Agnes, I. Albuquerque, T. Alexander, A. Alton, D. Asner, H. Back, B. Baldin, K. Biery, V. Bocci et al., J. Phys. Conf. Ser. 798, 012109 (2017).

[46] C. E. Aalseth et al., Eur. Phys. J. Plus 133, 131 (2018).

[47] P. Cushman et al., in Proceedings, Snowmass (2013).

[48] J. Aalbers et al. (DARWIN Collaboration), J. Cosmol. Astropart. Phys. 11 (2016) 017.

[49] R. Agnese et al. (SuperCDMS Collaboration), Phys. Rev. D 95, 082002 (2017).

[50] P. Agnes et al. (DarkSide Collaboration), Phys. Rev. Lett. 121, 081307 (2018).
[51] P. Agnes et al. (DarkSide Collaboration), Phys. Rev. D 98, 102006 (2018).

[52] E. Aprile et al. (XENON Collaboration), Phys. Rev. Lett. 119, 181301 (2017).

[53] E. Aprile et al. (XENON Collaboration), Phys. Rev. Lett. 121, 111302 (2018).

[54] D. S. Akerib et al. (LUX Collaboration), Phys. Rev. Lett. 118, 021303 (2017).

[55] X. Cui et al. (PandaX-II Collaboration), Phys. Rev. Lett. 119, 181302 (2017).

[56] E. Aprile et al. (XENON Collaboration), Phys. Rev. D 94, 092001 (2016); 95, 059901(E) (2017).

[57] E. Aprile et al. (XENON Collaboration), J. Cosmol. Astropart. Phys. 04 (2016) 027.

[58] D. S. Akerib et al. (LUX-ZEPLIN Collaboration), arXiv: 1802.06039 [Phys. Rev. D (to be published)].

[59] A. Heger, A. Friedland, M. Giannotti, and V. Cirigliano, Astrophys. J. 696, 608 (2009).

[60] A. Odrzywolek and A. Heger, Acta Phys. Pol. B 41, 1611 (2010), http://inspirehep.net/record/870759.

[61] W. C. Haxton, R. G. Hamish Robertson, and A. M. Serenelli, Annu. Rev. Astron. Astrophys. 51, 21 (2013).

[62] N. Grevesse and A. J. Sauval, Space Sci. Rev. 85, 161 (1998).

[63] M. Asplund, N. Grevesse, A. J. Sauval, and P. Scott, Annu. Rev. Astron. Astrophys. 47, 481 (2009).

[64] A. C. Hayes and P. Vogel, Annu. Rev. Nucl. Part. Sci. 66, 219 (2016).

[65] T. Araki et al., Nature (London) 436, 499 (2005).

[66] G. Bellini et al. (Borexino Collaboration), Phys. Lett. B 687, 299 (2010).

[67] Y. Huang, V. Chubakov, F. Mantovani, R. L. Rudnick, and W. F. McDonough, arXiv:1301.0365.

[68] D. Z. Freedman, D. N. Schramm, and D. L. Tubbs, Annu. Rev. Nucl. Part. Sci. 27, 167 (1977).

[69] R. H. Helm, Phys. Rev. 104, 1466 (1956).

[70] J. Erler and M. J. Ramsey-Musolf, Phys. Rev. D 72, 073003 (2005).

[71] K. Nakamura, S. Horiuchi, M. Tanaka, K. Hayama, T. Takiwaki, and K. Kotake, Mon. Not. R. Astron. Soc. 461, 3296 (2016).

[72] C. Babusiaux et al. (Gaia Collaboration), Astron. Astrophys. 616, A10 (2018).

[73] F. W. Chatys, T. R. Bedding, S. J. Murphy, L. L. Kiss, D. Dobie, and J. E. Grindlay, Mon. Not. R. Astron. Soc. 487, 4832 (2019).

[74] G. M. Harper, A. Brown, and E. F. Guinan, Astron. J. 135, 1430 (2008).

[75] D. S. Akerib et al. (LZ Collaboration), arXiv:1509.02910. 